\title{
Kineticand thermodynamic aspects of flotation beneficiation of polymetallic raw materials
}

\author{
T.D. Kalmykova, V.V. Kuznetsov \\ Mineral Processing Department, Mineral Raw Material Processing Faculty, Saint Petersburg Mining \\ University, Russia
}

\begin{abstract}
The article presents the results of the development of a methodological approach to the analysis of kinetic and thermodynamic parameters for improving the efficiency of flotation processing of polymetallic raw materials. The kinetic dependences of flotation, the hydrophobizing ability of butyl potassium xanthogenate, and the effect of reagents on preliminary mechanical activation were studied, and flotation modeling with and without an oxidizer was performed. The best results of convergence with the kinetic dependences of flotation are shown by the Beloglazov equation, the Kelsall and the modified Kelsall models. Technological indicators of sulfide flotation in the presence of oxidants are higher, which can be explained by the intensive oxidation of the formed transient ions on the surface of minerals, which contributes to the alignment of the flotation properties of the surface.It is possible to integrate the proposed research methods into technological schemes in order to optimize technological indicators and increase profits.
\end{abstract}

\section{Introduction}

Over time, the quality of processed ores and the content of metals in them continuously decrease. Over the past 20 years, the content of non-ferrous metals in ores has decreased by 1.3-1.5 times, the share of hard-to-enrich ores has increased to $40 \%$ of the total mass of raw materials received for processing.

Increasingly stringent requirements for the complex use of raw materials on the background of depletion of known balance deposits of non-ferrous and noble metals requires the use of new approaches in the enrichment of these raw materials [1].

To optimizetechnological indicators and increase the economic efficiency of processing polymetallic ores, it is necessary to use the kinetic and thermodynamic dependencies of flotation ore enrichment at the stage of designing technological schemes.

Kinetic studies and the distribution of minerals by floatability are necessary both for assessing ore enrichment at the initial stage and at the stage of a more detailed study.The study of flotation kinetics can be used to determine the time required for flotation.

The presence of an oxidized film on the surface of minerals changes their wettability characteristics, whichaffects the technical indicators of the flotation process. Effects occurring on the surface of minerals during oxidationcan be used for selective extraction of each sulfide and selection of the reagent mode for both grinding and flotation [2]. 
Due to the oxidation of xanthogenate to insoluble dixanthogenide in the process of sulfide flotation, the sorption form on the surface of the mineral may change.

The purpose of this study is thedevelopmentofa methodological approach to analyzingkinetic and thermodynamic parameters for improving the efficiency of flotation processing of polymetallic raw materials. To achieve this goal, the following tasks were set:

- Determine the zone of optimal values of the redox potential of the pulp for selective separation of minerals by thermodynamic modeling;

- Perform experimental studies to find the kinetic dependencies of the flotation process;

- Determine the dependence of the extraction of various minerals depending on the oxidizer;

- Compare the extraction values for flotation in the presence and absence of an oxidizer.

The kinetics of the flotation process is characterized by the dependence of mineral extraction on time.Based on the results of flotation kinetics analysis, it is possible,forexample,to justify the optimal distribution of foam removal along the flotation front,compare and evaluate the floatability of different minerals in different conditions,identify the effect of certain flotation factors.It is necessary to distinguish the kinetics of flotation from the kinetics of a chemical reaction. The chemical reaction constant evaluates the probability of conversion, while the constant of flotation kinetics determines, generally speaking, the process of fixing and removal of mineral particles.

All kinetic models can be divided into fundamental,i.e. based on the physical and chemical aspects of the flotation act, and empirical, obtained for specific data by regression processing [3]

In practice, the first-order kinetic equation (the model of K. F. Beloglazov) is mostly used [4]. Many researchers have proposed more precise models to describe the flotation kinetics of various minerals (Fig. 1).

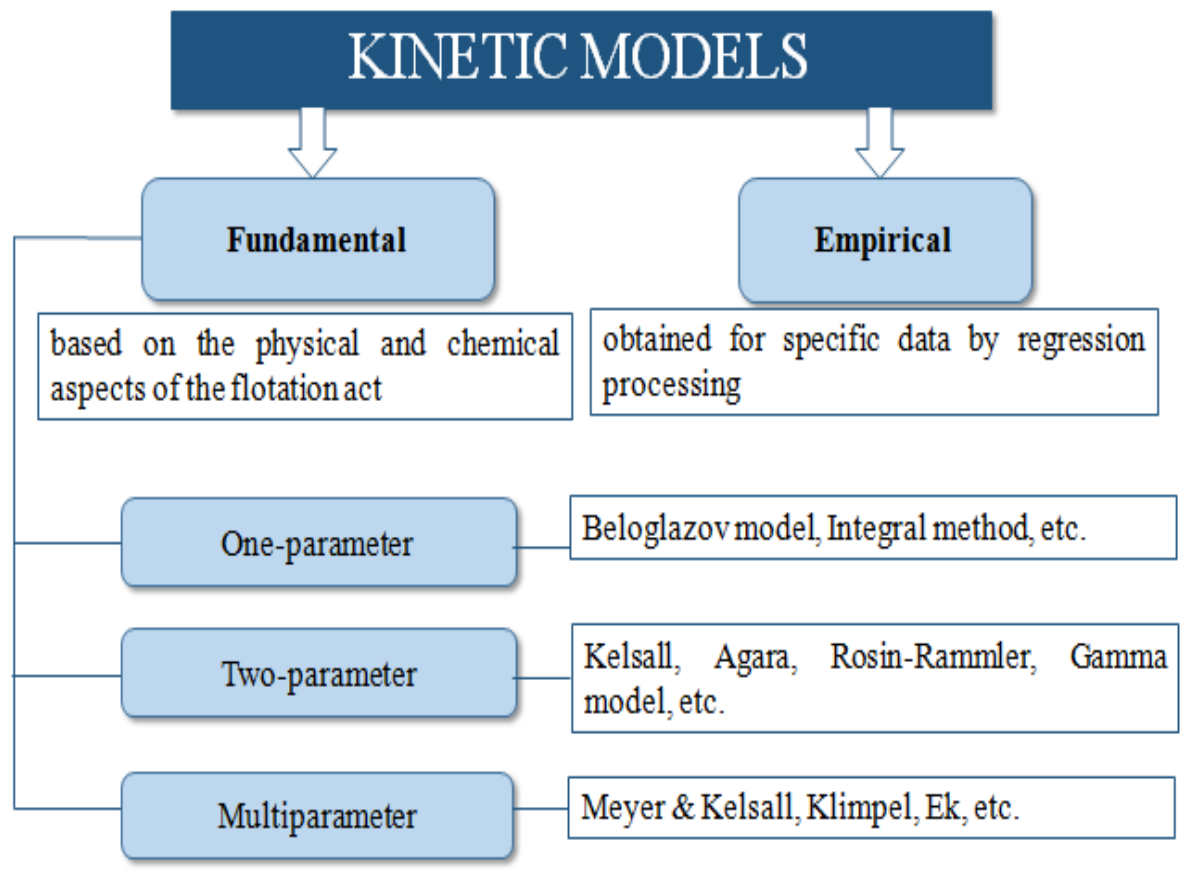

Fig. 1.Kinetic models of flotation beneficiation. 
The selection of the Eh and $\mathrm{pH}$ range is necessary for selective flotation of minerals while keeping the stability of their oxidation process. Under these conditions, it is possible to select optimal concentrations of oxidants to improve the required technological indicators. The zone of stable existence of sulfate ions will be the optimal flotation zone of the raw material [5].

\section{Materials and methods}

Carbon-containing sulfide copper-zinc and gold-containing ores were selected as the objects of research.

Dictionem shales are grayish-black, black, and brown thinly layered carbonaceous rocks. This is alow-grade oil shale due toitshigh ash content.

The choice of shale as an object of research is due to the importance of this object as a source of strategic metals, such as precious, rare, non-ferrous metals, and trace elements $[6,7,8]$.

Copper-pyrite ores are an important industrial type of copper-sulfide ores. The main valuable components are copper, zinc, and sulfur.The development of these deposits is profitable due to the possibility of complex processing of raw materials, that is, the accompanyingextraction of components with copper (zinc, gold, silver, sulfur, iron), the cost of which is significantly higher than the cost of copper[9].

For sulfide gold-containing ores, resistanceisa characteristic feature. As a rule, goldcontaining sulfides (mainly pyrite and arsenopyrite) have a crystal structure that is poorly permeable to cyanide solutions.Most of the grains of native gold in them have thin and ultra-thin dimensions.More than $80 \%$ of the particles are less than 20 microns in size.The presence of sulfide and oxidized minerals of non-ferrous metals requires a special chemical treatment that is associated with the oxidation ofsulfides associated withgold $[5,10]$.

The composition of ores is shown in Figure 2.


Fig. 2.Mineralogical composition of the ores selected for experiments. 
To determine the kinetic dependences of flotation of selected ores, flotation enrichment of the selected raw materials was carried out on a flotation machine JK Flotation Test Batch Machine with subsequent X-ray fluorescence analysis of concentrates on a spectrometer EDX-7000 Shimadzu.

The scheme of experiments is shown in Figure 3.

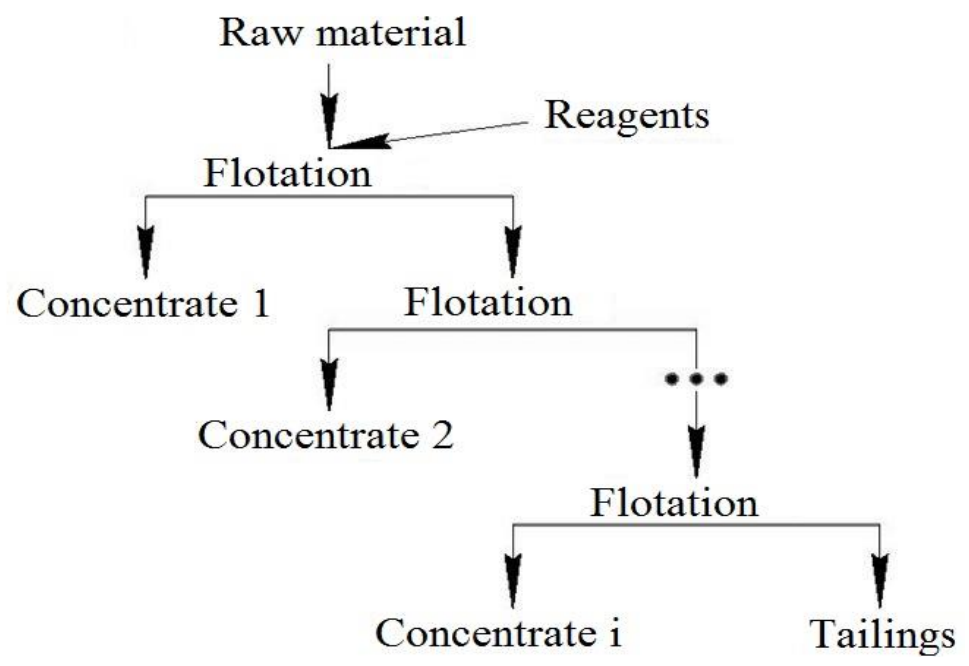

Fig. 3.The scheme of experiments.

The HSC Chemistry 9.0 program was used to evaluate the chemical reaction on the mineral surface through the formation of various products of this reaction. It is an environment for creating models for various types of basic operations from chemical processes to economic optimization.To achieve the set goals of the study, the capabilities of the program to create Pourbaixdiagramswere used.

\section{Resultsanddiscussion}

According to the analysis of the obtained data, it is difficult toconclude about the functional form of kinetic dependencies (Table 1).To determine the type of a flotation model, computer programs developed at the Department ofEnrichment of Mining University were used.The criterion for selecting suitable dependencies was the coefficient of determination $-\mathrm{R}^{2}$.

Table 1.Results of the study of kinetic dependencies.

\begin{tabular}{llllllll}
\hline \multicolumn{2}{l}{ Copper-zinc } & \multicolumn{3}{l}{ Gold-containing } & \multicolumn{3}{l}{ Carbonaceous } \\
\cline { 3 - 6 } & & Pyrite & \multicolumn{3}{l}{ Arsenopyrite } & \\
\hline $\begin{array}{l}\text { Flotation } \\
\text { time, } \mathrm{s}\end{array}$ & $\begin{array}{l}\text { Total ex- } \\
\text { traction, } \\
\%\end{array}$ & $\begin{array}{l}\text { Flotation } \\
\text { time, } \mathrm{s}\end{array}$ & $\begin{array}{l}\text { Total ex- } \\
\text { traction, } \%\end{array}$ & $\begin{array}{l}\text { Flotation } \\
\text { time, } \mathrm{s}\end{array}$ & $\begin{array}{l}\text { Total ex- } \\
\text { traction, } \%\end{array}$ & $\begin{array}{l}\text { Flotation } \\
\text { time, } \mathrm{s}\end{array}$ & $\begin{array}{l}\text { Total ex- } \\
\text { traction, } \%\end{array}$ \\
\hline 0.00 & 0 & 0.00 & 0.00 & 0.00 & 0.00 & 0.00 & 0.00 \\
30.00 & 12.28 & 30.00 & 4.63 & 30.00 & 1.24 & 60.00 & 12.50 \\
60.00 & 16.89 & 60.00 & 11.61 & 60.00 & 3.52 & 180.00 & 28.30 \\
120.00 & 34.96 & 120.00 & 15.56 & 120.00 & 4.94 & 420.00 & 42.30 \\
240.00 & 53.71 & 240.00 & 19.07 & 240.00 & 5.87 & 720.00 & 54.60 \\
360.00 & 60.60 & 360.00 & 22.21 & 360.00 & 6.41 & 1800.00 & 68.70 \\
720.00 & 68.51 & 720.00 & 24.83 & 720.00 & 6.87 & & \\
\hline
\end{tabular}


While using an integral method for determining the kinetic dependence of flotation enrichment of copper-zinc ore and gold-containing ore (arsenopyrite flotation), it was found that the Beloglazov equation has a maximum convergence coefficient (Table 2).

Table 2.Results obtained using the integral method.

\begin{tabular}{llllll}
\hline Equation & Coefficient & \multicolumn{2}{l}{ Typeofore } & \\
\cline { 3 - 5 } & & Copper- & \multicolumn{2}{l}{ Gold-containing } & Carbonaceous \\
\cline { 3 - 5 } & & zinc & Pyrite & Arsenopyrite & \\
\hline BeloglazovEquation & $\mathrm{R}$ & 0.99814 & 0.99056 & 0.99201 & 0.99625 \\
& $\mathrm{R} \infty$ & 0.70106 & 0.24831 & 0.06867 & 0.68700 \\
& $\mathrm{k}$ & 0.33927 & 0.46820 & 0.58946 & 0.14794 \\
\hline Order 0,5 & $\mathrm{R}$ & 0.93669 & 0.91818 & 0.88186 & 0.94661 \\
& $\varphi$ & 0.16678 & 0.08952 & 0.04556 & 0.09862 \\
& $\mathrm{~b}$ & 0.30939 & 0.22318 & 0.12674 & 0.33030 \\
\hline Order 1 & $\mathrm{R}$ & 0.97284 & 0.97554 & 0.95652 & 0.98933 \\
& $\varphi$ & 0.45505 & 0.36080 & 0.32905 & 0.40371 \\
& $\mathrm{~b}$ & -1.41880 & -2.21900 & -3.39840 & -1.70200 \\
\hline Order 1,5 & $\mathrm{R}$ & 0.98762 & 0.99007 & 0.97859 & 0.99853 \\
& $\varphi$ & 1.28100 & 1.54590 & 2.56760 & 1.74430 \\
& $\mathrm{~b}$ & 0.80141 & 1.52510 & 2.95300 & 0.87740 \\
\hline Order 2 & $\mathrm{R}$ & 0.99490 & 0.99494 & 0.98993 & 0.99929 \\
& $\varphi$ & 3.50840 & 6.17220 & 18.35370 & 7.36340 \\
& $\mathrm{~b}$ & 1.11490 & 3.51580 & 12.61250 & 1.22020 \\
\hline
\end{tabular}

When determining the kinetic dependence using the integral method for flotation of pyrite from sulfide ore and carbon-containing raw materials, it was found that the secondorder equation has the maximum convergence (see Table 2). An example of calculation for carbon-containing raw materials is shown in Figure 4.
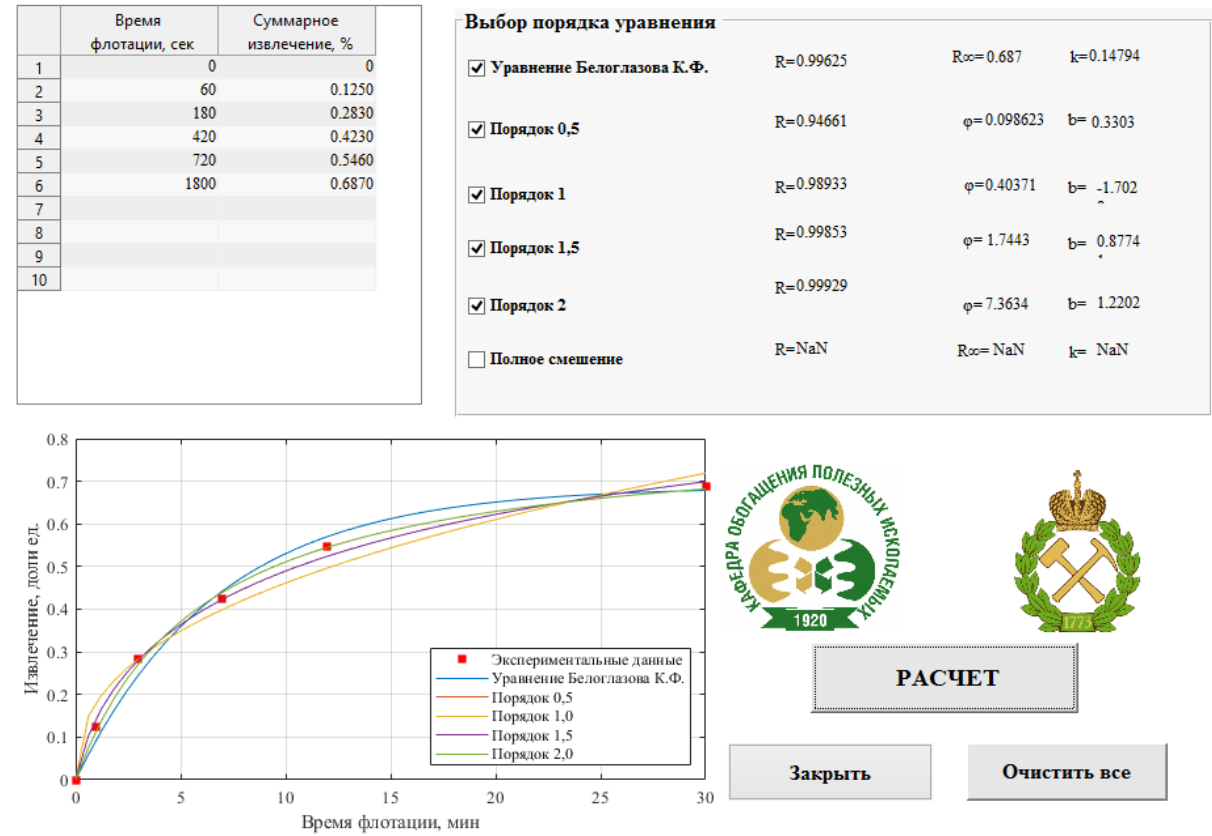

Fig.4.The example of using the program for calculating the kinetic dependence of the integral method for carbon-containing raw materials. 
Using the program to determine the constants of the most commonly used equations for copper-zinc ore showed the maximum coefficient of determination for the classical (Beloglazov) model, the Modified Kelsall model, and the Gamma model.Using the program to determine the constants of the most commonly used equations for carbonaceous ore showed that themodified Kelsall model has the maximum convergence with experimental data (Table 3).

Table 3.Results obtained in the analysis of equations.

\begin{tabular}{llllll}
\hline Equation & \multirow{2}{*}{ Coefficient } & \multicolumn{2}{l}{ Typeofore } & & \\
\cline { 4 - 5 } & & Copper- & \multicolumn{2}{l}{ Gold-containing } & Carbonaceous \\
\cline { 4 - 5 } & & zinc & Pyrite & Arsenopyrite & \\
\hline BeloglazovEquation & $\mathrm{R}$ & 0.99814 & 0.99056 & 0.99211 & 0.996292 \\
& $\mathrm{R} \infty$ & 0.70106 & 0.24831 & 0.06867 & 0.679 \\
& $\mathrm{k}$ & 0.33927 & 0.4682 & 0.058992 & 0.153487 \\
\hline KlimpelEquation & $\mathrm{R}$ & 0.99566 & 0.98992 & 0.98787 & 0.996834 \\
& $\mathrm{R} \infty$ & 0.80643 & 0.26337 & 0.074034 & 0.745606 \\
& $\mathrm{k}$ & 0.6309 & 1.0617 & 1.2606 & 0.321071 \\
\hline KelsallEquation & $\mathrm{R}$ & 0.99607 & 0.99545 & 0.99374 & 0.995899 \\
& $\varphi$ & 0.49757 & 0.81389 & 0.093864 & 0.679795 \\
& $\mathrm{k}_{\mathrm{f}}$ & 0.5 & 0.74771 & 0.70902 & 0.5 \\
& $\mathrm{k}_{\mathrm{s}}$ & 0.042306 & 0.0067261 & 0.0063787 & 0.027224 \\
\hline ModifiedKelsallequation & $\mathrm{R}$ & 0.99614 & 0.99556 & 0.99374 & 0.999867 \\
& $\mathrm{R} \infty$ & 0.70106 & 0.27823 & 0.38567 & 0.701533 \\
& $\mathrm{k}_{\mathrm{f}}$ & 0.5 & 0.85305 & 0.70918 & 1.034673 \\
& $\mathrm{k}_{\mathrm{s}}$ & 0.33927 & 0.11771 & 0.0018654 & 0.109951 \\
& $\varphi$ & 1 & 0.43401 & 0.84097 & 0.830996 \\
\hline Gammamodel & $\mathrm{R}$ & 0.99614 & 0.99496 & 0.99305 & 0.999354 \\
& $\mathrm{R} \infty$ & 0.70111 & 0.27447 & 0.06867 & 1 \\
& $\lambda$ & 9596.1085 & 2.1164 & 7.2799 & 2.983695 \\
& $\mathrm{P}$ & 3255.6165 & 1.2121 & 4.7946 & 0.476937 \\
\hline Fullymixedmodel & $\mathrm{R}$ & 0.95534 & 0.98556 & 0.98673 & 0.911197 \\
& $\mathrm{R} \infty$ & 0.6851 & 0.24831 & 0.072536 & 0.679 \\
& $\mathrm{k}$ & 1 & 1 & 1 & 1 \\
\hline
\end{tabular}

An example of calculation for copper-zinc ore is shown in Figure 5. 


\begin{tabular}{|c|c|c|}
\hline & $\begin{array}{c}\text { Flotation } \\
\text { Time [s] }\end{array}$ & $\begin{array}{l}\text { Cumulative } \\
\text { Recovery [ ] }\end{array}$ \\
\hline 1 & 0 & 0 \\
\hline 2 & 30 & 0.1228 \\
\hline 3 & 60 & 0.1689 \\
\hline 4 & 120 & 0.3496 \\
\hline 5 & 240 & 0.5371 \\
\hline 6 & 360 & 0.6060 \\
\hline 7 & 720 & 0.6851 \\
\hline 8 & & \\
\hline 9 & & \\
\hline 10 & & \\
\hline
\end{tabular}

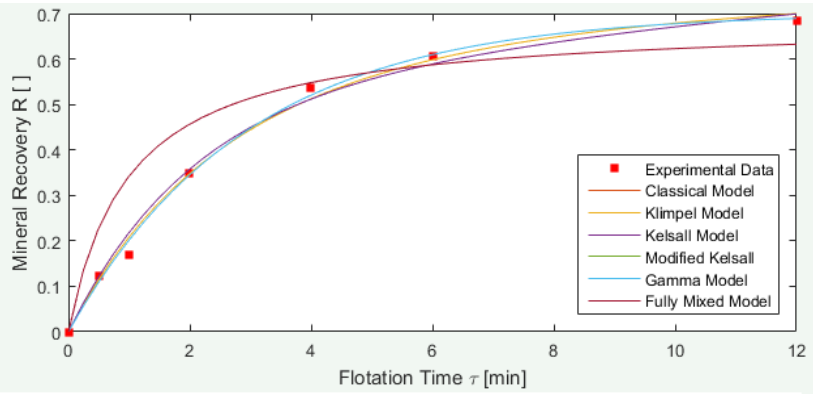

\begin{tabular}{|lll} 
Flotation Model Selection & & \\
$\square$ Classical & $\mathrm{R}=0.99814$ & $\mathrm{R}_{\infty}=0.70106$ \\
$\square$ Klimpel & $\mathrm{R}=0.99566$ & $\mathrm{R}_{\infty}=0.80643$ \\
$\square$ Kelsall & $\mathrm{R}=0.99607$ & $\varphi=0.49757$ \\
$\square$ Modified Kelsall & $\mathrm{R}=0.99814$ & $\mathrm{R}_{\infty}=0.70106$ \\
$\square$ Gamma Model & $\mathrm{R}=0.99814$ & $\mathrm{R}_{\infty}=0.70111$ \\
$\square$ Fully Mixed & $\mathrm{R}=0.95534$ & $\mathrm{R}_{\infty}=0.6851$ \\
& & \\
& & \\
& & \\
& & \\
\end{tabular}

$\mathrm{k}=0.33927$

$\mathrm{k}=0.6309$

$k_{\mathrm{f}}=0.5$

$\mathrm{k}_{\mathrm{f}}=0.5$

$\lambda=9596.1085$

$\mathrm{k}=1$

$$
\begin{aligned}
& \mathrm{k}_{\mathrm{s}}=0.042306 \\
& \mathrm{k}_{\mathrm{s}}=0.33927 \\
& \mathrm{P}=3255.8165
\end{aligned} \quad \varphi=1
$$

Fig. 5.Using the program to select the most appropriate equation for copper-zinc ore.

When using the program to determine the constants of the most commonly used equations for gold-containing ore, it was found that the Kelsall equation has maximum convergence with the empirical dependence for both pyriteand arsenopyrite flotation (see Table 3).

Analysis of the hydrophobizing ability of the primary collector for the flotation of sulfide of potassium butyl xanthate was carried out in two stages: the wetting angle at the mineral-water-air boundary was determined without treating the mineral surface with a reagent and with its pretreatment by a collector, then the floatability parameter was calculated for both cases (Fig. 6).
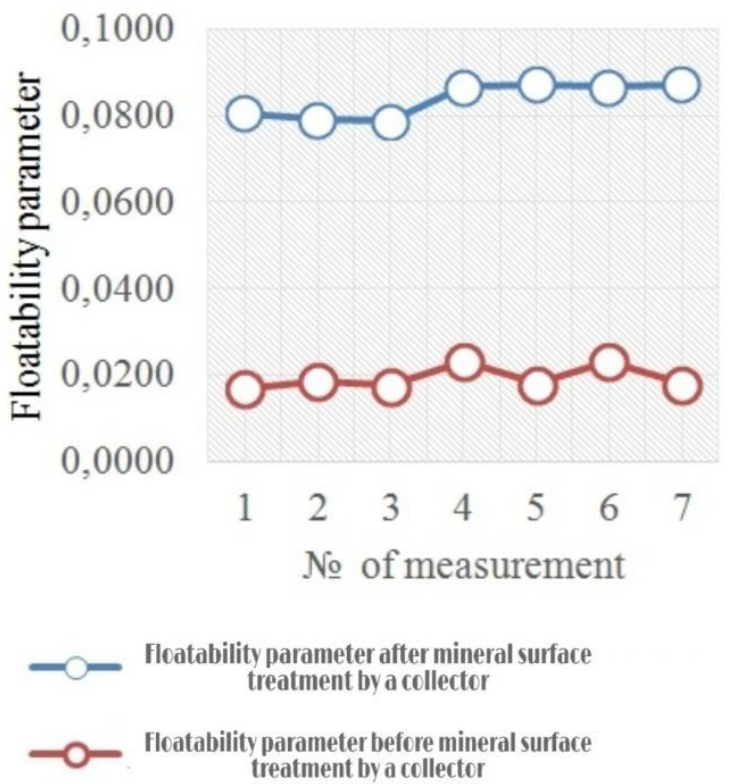

Fig. 6. Floatability parameter before and after mineral surface treatment by the collector. 
The floatability parameter F can be calculated using the formula:

$$
F=\sigma_{\mathrm{g}-\mathrm{l}}(1-\cos \theta)
$$

$\sigma_{\mathrm{g}-1}$ is the surface tension at the gas-liquid interface; $\cos \theta$ is the cosine of the wetting angle of the three-phase contact perimeter [4].

Based on the data obtained, it can be concluded that the pyrite surface is hydrophobized,as the obtained values of the wetting angle are higher than $90^{\circ}$, the floatability parameter is increased.

The formation of sulfate ions will be a factor of optimal use of oxidizers in the flotation process.For stable oxidation of the surface of minerals, it is necessary to select the appropriate range of Eh and $\mathrm{pH}$.

The zone of the existence of sulfate ions is characterized by the highest probability of oxidation of the pyrite surface in gold-containing ore $[11,12]$.

When comparing the results of flotation simulation with the use of an oxidizer and without it, an increase in the zone of the existence of sulfate ions is observed by $7.16 \%$ (from 1.72 to 1.85 ) (Fig. 7).
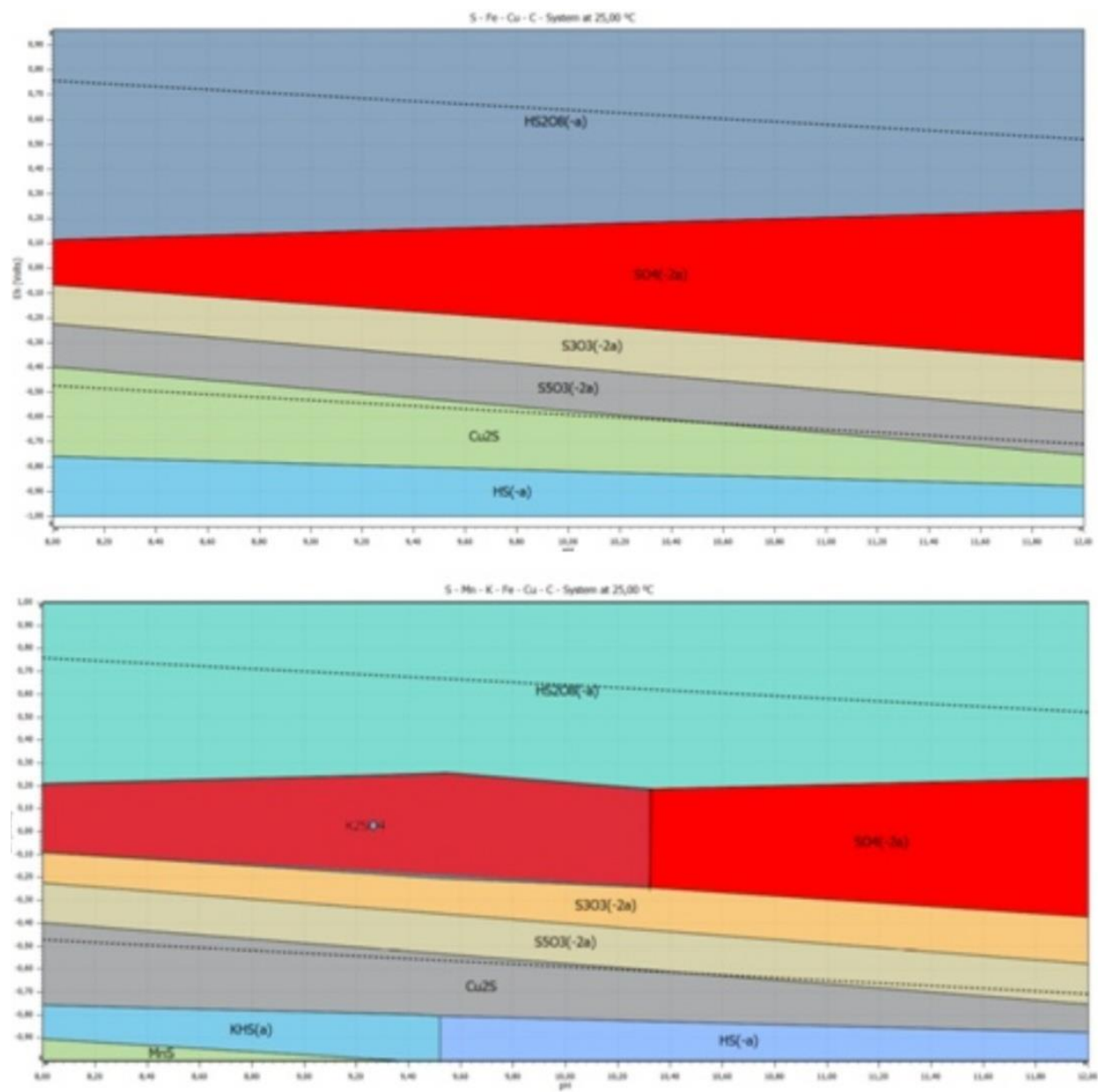

Fig. 7.Model of the flotation process: top-without the use of an oxidizer; bottom-with the use of an oxidizer. 
Based on the obtained data, we can conclude about the influence of preliminary mechanical activation of copper-zinc sulfide ores on the technological indicators of their flotation enrichment (Fig. 8).

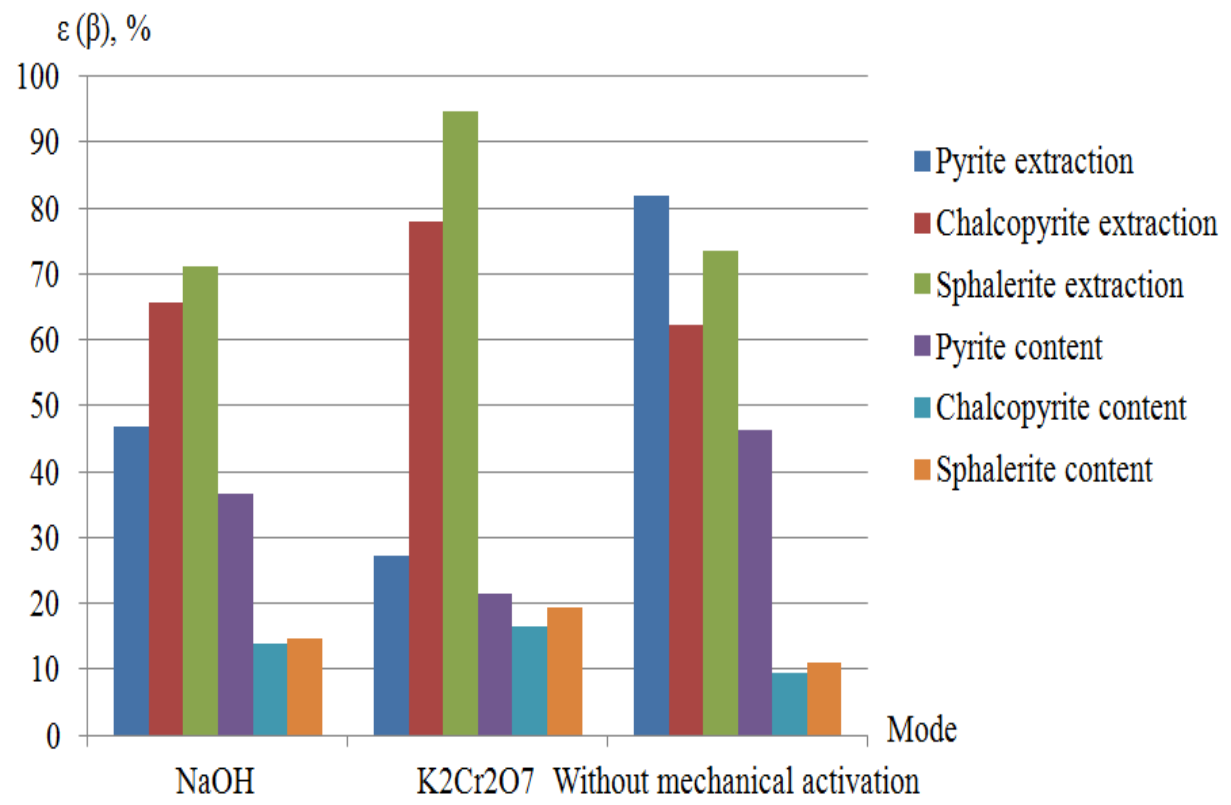

Fig. 8.Estimation of the effect of preliminary mechanical activation of copper-zinc ores.

When adding potassium bichromate as a dispersant, the content of chalcopyrite and sphalerite in the collective concentrate is maximum, which is a positive factor in flotation enrichment. At the same time, the extraction of pyrite is minimal, which can be used at the stage of selective flotation, since the extraction of pyrite in selective copper-zinc concentrates is undesirablesince during their further pyrometallurgical processing, a large amount of sulfur contained in iron sulfides is released [13].

\section{Conclusion}

According to the data obtained in the study of kinetic dependencies of various raw materials, the best convergence results are shown by the Beloglazov equation, the Kelsall model, and themodified Kelsall model.

Technological indicators of sulfide flotation in the presence of oxidants are higher,whichcan be explained by the intense oxidation of the transition ions formed on the surface of minerals, which helps to align the flotation properties of the surface.

It is possible to integrate the proposed research methods into technological schemes to optimize technological indicators and increase profits.

The workwassupported by the Russian Science Foundation (project No. 19-17-00096).We want to express our gratitude to Prof. Tatyana N.Aleksandrova, Dr. of Engineering, Head of the Mineral Processing Department of Mining University. 


\section{Referenses}

1. N. K. Algebraistova The technology of enrichment of non-ferrous metal ores: lecture notes, (Krasnoyarsk: IPK NFU. 2009)

2. V. A. Glembotskiy Fundamentals of physical chemistry of flotation processes, (Moscow: Nedra, 1980)

3. M. Safari, D. Deglon An attachment-detachment kinetic model for the effect of energy input on flotation. Minerals Engineering, 117, 8-13 (2018)

4. K. F. Beloglazov Regularities of flotation enrichment. Current state and prospects of flotation theory development, (Moscow: Metallurgizdat, 1947)

5. T. N. Aleksandrova, A. O. Romashev, D. N. Semenikhin Mineral and technological aspects and promising methods for intensifying the enrichment of gold-containing sulfide ore. Metallurgist, 4, 53-59 (2015)

6. T. N. Aleksandrova, N. V. Nikolaeva, A. O. Romashev, A. V. Aleksandrov Noble and rare metals in caustobioliths and prospects of their recovery. Journal of Mining Science, 6, 1254-1261 (2015)

7. T. N. Aleksandrova, A. V. Afanasova Fine-dispersed particles of noble metals in sulphide carbonaceous ores and its beneficiation prospects. 29th International Mineral Processing Congress, 2368-2376 (2018)

8. A. V. Afanasova, T. N. Aleksandrova, N. V. Nikolaeva Processing of carbonaceous ores containing ultra-dispersed metals with using high-frequency current. International Multidisciplinary Scientific GeoConference Surveying Geology and Mining Ecology Management, SGEM 2018, 119-124 (2018)

9. A. A. Abramov The technology of processing of non-ferrous metal ores: textbook. manual for universities: in 2 books, (Moscow: Moscow State University Press, 2005)

10. L. V. Shumilova Analysis of the reasons for the resistance of ores with dispersed gold in cyanidation. Mining information and analytical bulletin (scientific and technical journal), 6, (2009)

11. T. N. Aleksandrova, V. V.Kuznetsov, A. V. Chanturia Increasing the efficiency of sulfide flotation taking into account thermodynamic modeling data. Materials of the international scientific Symposium "Plaksin readings-2018" in the framework of IMPC 2018, (2018)

12. T. N. Alexandrova, D. N. Semenikhin, V. A. Potemkin Evaluation of the efficiency of flotation separation by the method of interpretation of modeling data; Current trends in the enrichment of natural and man-made raw materials of various mineral composition. Mining informational and analytical bulletin, 3-11 (2018)

13. V. L. Trushko, R. E. Dashko, V. B. Kuskov, A. V. Kornev, A. S. Klyamko Preparation of iron ore raw materials for metallurgical processing. Zapiski Gornogo Instituta, 194, $120-123(2011)$ 